\title{
Joint AMC and Packet Fragmentation for Error Control Over Fading Channels
}

\author{
Ruonan Zhang, Student Member, IEEE, and Lin Cai, Member, IEEE
}

\begin{abstract}
Error control is critical for wireless networks to combat channel fading and ensure efficient resource utilization. Adaptive modulation and coding (AMC) in the physical (PHY) layer and packet fragmentation and automatic repeat request (ARQ) in the link layer are widely used error-control mechanisms. However, how to jointly optimize them in both layers for high-rate wireless networks is still open. In this paper, using the WiMedia ultrawideband (UWB) networks as an example, we first develop a general analytical framework to quantify the link delay and loss performance considering the channel fading, the joint errorcontrol mechanisms, and the arbitrary reservation-based media access control (MAC) protocol. Second, we introduce a cross-layer design to optimize the PHY-layer AMC and the link-layer packet fragmentation and propose a joint-adaptation mechanism that is simple to implement and has near-optimal performance. Numerical results reveal that fragmentation has a greater impact than AMC on the delay and loss performance for marginal links and that the proposed joint-adaptation strategy is efficient for high-rate wireless networks.
\end{abstract}

Index Terms-Adaptive modulation and coding, automatic repeat request, error control, fading channels, packet fragmentation, queueing analysis, reservation-based medium access control.

\section{INTRODUCTION}

$\mathbf{T}$ HE third-generation/fourth-generation cellular systems, IEEE 802.11 wireless local area networks, and IEEE 802.15.3a wireless personal area networks (WPANs) have evolved into support high-data-rate multimedia services. As wireless channels are error prone and broadcast in nature, both error control and media access control (MAC) are critical for resource utilization and quality-of-service (QoS) provisioning. Combining them further complicates the network performance study and protocol optimization.

To provide reliable data delivery over the fading channels and enhance bandwidth efficiency, various error-control mechanisms have been proposed. Adaptive modulation and coding (AMC) in the physical (PHY) layer can maintain the target bit error rate (BER) by changing the transmission mode (TM) in

Manuscript received October 23, 2009; revised January 5, 2010; accepted February 8, 2010. Date of publication March 29, 2010; date of current version July 16, 2010. This work was supported in part by grants from the Natural Sciences and Engineering Research Council of Canada. This paper has been presented in part at the IEEE Global Telecommunications Conference, New Orleans, LA, December 2008. The review of this paper was coordinated by Prof. C. Lin.

The authors are with the Department of Electrical and Computer Engineering, University of Victoria, Victoria, BC V8W 3P6, Canada (e-mail: rzhang@ece.uvic.ca; cai@ece.uvic.ca).

Color versions of one or more of the figures in this paper are available online at http://ieeexplore.iee.org.

Digital Object Identifier 10.1109/TVT.2010.2046758 every frame or a burst of frames, according to the channel condition. In the link layer, the packet fragmentation and automatic repeat request (ARQ) schemes are employed, which are particularly important for marginal links (links with small SNR budget). A packet from the upper layer, called MAC service data unit (MSDU), ${ }^{1}$ may be fragmented by the sender and then reassembled at the receiver. The fragments are delivered using the ARQ scheme, such as the delayed acknowledgment (Dly-ACK) ARQ (which will be described in Section III-C). In the following, we denote AMC in the PHY layer and fragmentation and ARQ in the link layer together as the joint error-control mechanisms.

On the other hand, the MAC protocol coordinates the network nodes to share the medium, and it also affects the QoS support. Due to the opportunistic access nature, the contentionbased MAC cannot ensure QoS, and the prioritized contentions may lead to the starvation of low-priority flows [1]. Consequently, the reservation-based MAC protocols have been adopted in emerging standards due to better QoS support, for example, the distributed reservation protocol (DRP) in the WiMedia ECMA-368 [2] and the channel time-allocation specification in IEEE 802.15.3a [3] for ultrawideband (UWB) WPANs. However, different from the traditional time-division multiplexing access (TDMA) protocol where one reserves a number of continuous slots per scheduling period, with a general distributed reservation-based MAC such as the DRP, a node may reserve multiple noncontinuous slots arbitrarily distributed in a scheduling period.

To ensure QoS support, we need to quantify the network performance such as the packet loss and delay, considering the wireless channel variation, error-control mechanisms, and scheduling. In this paper, using the ECMA-368 standard as an example, we investigate AMC in the PHY layer and Dly-ACK ARQ, packet fragmentation, and DRP MAC in the link layer for UWB networks with fading channels.

The main contributions of this paper are threefold: First, we propose a Markov model to quantify the queuing process of the fragments of MSDUs at the sender's buffer. Second, we study the transmission process of a fragmented MSDU delivered by Dly-ACK over the fading channel. Thus, a complete analytical framework to quantify the packet drop rate (PDR) and delay is developed. Third, a cross-layer optimization problem for AMC and fragmentation is formulated, and a feasible suboptimal joint-adaptation strategy is proposed. Different from the previous works studying AMC and ARQ, we consider two more

\footnotetext{
${ }^{1}$ The MSDUs refer to the packets from the upper layer to the MAC layer to transmit. The terms MSDU and packet are interchangeable in this paper.
} 
issues, namely, the arbitrary reservation pattern in scheduling and the estimation errors in channel state information (CSI). Furthermore, this paper is the first to theoretically study the queuing and transmission processes of fragmented packets and compare the effects of AMC and fragmentation on network performance.

We have the following key observations: 1) The PHY-layer $\mathrm{AMC}$ and the link-layer fragmentation can both improve the bandwidth utilization and link performance. However, fragmentation can also improve the queuing behavior of the buffer by allowing packets to be partially delivered, which further reduces the queuing delay and buffer overflow probability. 2) The fragmented packets may need more transmission opportunities to be completely delivered, resulting in larger transmission delay. However, the transmission delay increment is marginal compared with the reduced queuing delay. Therefore, fragmentation might be more important than AMC in terms of ensuring PDR and delay. 3) The proposed suboptimal joint-adaptation strategy can effectively combat the channel fading and improve the link throughput and delay, and it is easy to implement. Although we use the UWB WPANs as an example, the error-control technologies and the arbitrary reservation-based scheduling are general and widely adopted. Therefore, the proposed analytical framework and the joint-adaptation strategy are ready to be extended to other wireless systems, such as the millimeterwave-based WPANs.

The rest of this paper is organized as follows: We briefly survey the related works in Section II and overview the ECMA-368 standard and UWB channel model in Section III. The queuing model for the sender's buffer is presented in Section IV, and the transmission process of a fragmented packet is studied in Section V. Section VI evaluates the throughput by combining $\mathrm{AMC}$ and fragmentation and proposes the suboptimal jointadaptation mechanism. Section VII validates the analytical models by simulations and compares the performance of three different error-control mechanisms, followed by the concluding remarks in Section VIII.

\section{RELATED WORK}

The performance of traditional TDMA has extensively been modeled (e.g., [4] and [5]). Wu et al. [6] analyzed the queue distribution for the DRP and evaluated the effect of the reservation pattern on the link delay, assuming an ideal channel condition without transmission error. Liu et al. [7] studied the DRP performance with bursty arrivals and arbitrary reservation slot allocation over an indoor UWB shadowing channel. In these works, the error-control mechanisms, which have significant impact on the delay and throughput, have not been considered.

Dly-ACK, which is defined in the IEEE 802.15.3a [3] and ECMA-368 [2] standards, has recently drawn attention. The link throughput using Dly-ACK was derived by considering the effective transmitting time of the payload in [8]. In [9], the delay performance was analyzed, assuming independent transmission errors. An analytical model for Dly-ACK over a wireless Rayleigh-fading channel was developed in [10], which illustrated that the correlation between transmission errors affects link performance.

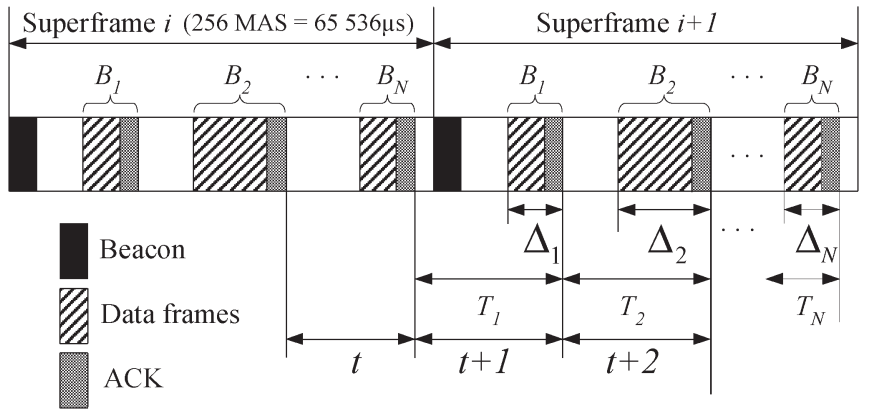

Fig. 1. MAS reservation in a superframe.

Many recent works have studied the cross-layer design combining AMC and ARQ for low-rate wireless networks using traditional TDMA MAC (e.g., [11] and [12]). By using a separate feedback channel, the CSI is usually assumed accurate. However, in practical wireless networks with channel-access scheduling, the transmitter has to use the CSI obtained from the current frame exchange to determine the TM for the next transmission opportunity, which may not be accurate due to channel variations and the relatively long access interval. Therefore, the performance of AMC can be degraded.

To the best of our knowledge, no previous work has studied the link-layer packet fragmentation, such as the queuing and transmission processes of fragmented packets, which have significant different throughput and delay properties. Furthermore, the link performance of the joint error-control mechanisms (AMC, ARQ, and packet fragmentation) combining with the arbitrary reservation-based MAC and the fading channel has not been reported, which motivates this work.

\section{Protocol Description And System Model}

\section{A. Superframe Structure and DRP}

The basic timing structure for ECMA-368 is a superframe. As depicted in Fig. 1, the superframe duration of $T_{\mathrm{SF}}=$ $65536 \mu \mathrm{s}$ is divided into 256 media access slots (MASs). An MAS lasts for $256 \mu \mathrm{s}$ and is the minimum time unit for reservation. Each superframe starts with a beacon period, where the availability information element (IE) indicates the current utilization of MASs in the superframe. Then, in the data transfer period, users communicate with each other through contentionor reservation-based channel access.

Using the DRP, each node negotiates with its target to reserve MASs according to its traffic load, QoS requirement, and availability of the MASs. To reduce the delay variation, it is desired to reserve evenly spaced time blocks. However, because the reservation is performed in a distributed manner, the reserved MASs of a source-destination pair can arbitrarily be located in one superframe, as shown in Fig. 1. A reservation block $(R B)$ is one or multiple continuous MAS(s) reserved by the same user. A user is said to be in service during its RBs, and on vacation otherwise. The duration between two consecutive RBs of the user is called vacation time. Each RB and the preceding vacation time together is named a reservation slot $(R S)$.

We focus on a single pair of users that have reserved $N$ RBs in each superframe, indexed by $n(n=1,2, \ldots, N)$ and with 
TABLE I

TMS IN MB-OFDM

\begin{tabular}{l|c|c|c|c|c|c|c}
\hline \hline$c$ & $\begin{array}{c}\text { Data Rate } \\
(\mathrm{Mbps})\end{array}$ & $N_{I B P 6 S}$ & $\begin{array}{c}\text { SNR interval } \\
{\left[\gamma_{c-1}, \gamma_{c}\right)(\mathrm{dB})}\end{array}$ & $\begin{array}{c}\text { Optimal Payload } \\
L_{c}^{*}(\text { bytes })\end{array}$ & $\begin{array}{c}\varepsilon_{0} \\
\left(\times 10^{-6}\right)\end{array}$ & $\begin{array}{c}\varepsilon_{w} \\
\left(\times 10^{-4}\right)\end{array}$ & $\begin{array}{c}\varepsilon_{b} \\
\left(\times 10^{-7}\right)\end{array}$ \\
\hline 1 & 160 & 300 & {$[6.15,7)$} & $820 \sim 2620$ & 8.74 & 2.55 & - \\
2 & 106.7 & 200 & {$[4.45,6.15)$} & $870 \sim 3640$ & 6.37 & 2.16 & 1.57 \\
3 & 80 & 150 & {$[2.85,4.45)$} & $370 \sim 2730$ & 15.9 & 2.95 & 3.64 \\
4 & 53.3 & 100 & {$[1,2.85)$} & $270 \sim 2580$ & 7.33 & - & 2.66 \\
\hline \hline
\end{tabular}

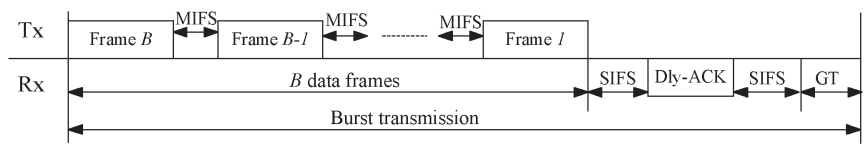

(a)

\begin{tabular}{|l|c|c|c|}
\hline bits: b15 & b14 & b13 - b3 & b2 - b0 \\
\hline Reserved & More Fragments & Sequence Number & Fragment Number \\
\hline
\end{tabular}

(b)

Fig. 2. Link-layer error control. Dly-ACK and fragmentation [2]. (a) Timing of the burst transmission of Dly-ACK. (b) Fragmentation sequence control field.

the duration of $\Delta_{n}$, as shown in Fig. 1. The duration of the $n$th $\mathrm{RS}$ is denoted by $T_{n}$.

\section{B. $A M C$}

ECMA-368 adopts the multiband orthogonal frequencydivision multiplexing (MB-OFDM) technology in the PHY layer. By puncturing the $R=1 / 3$ convolutional codes and using time/frequency domain spreading, various TMs with different data rates and coding and diversity gains are realized. Suppose that the wireless system can support $C$ TMs and denote $\mathcal{M}_{c}(c=1,2, \ldots, C)$ as the $c$ th TM. The range of the received SNR $\gamma$ is partitioned into $C$ fading intervals $\Gamma_{c}=$ $\left[\gamma_{c}, \gamma_{c-1}\right)$, and TM $\mathcal{M}_{c}$ is used when $\gamma \in \Gamma_{c}$ such that the target BER is maintained, which is denoted by $\varepsilon_{0}$.

The transmission time of a PHY-layer convergence protocol (PLCP) frame with payload size of $L$ bytes using TM $\mathcal{M}_{c}$ is [13]

$$
T_{F}\left(L, \mathcal{M}_{c}\right)=6 \times\left\lceil\frac{8 L+38}{N_{\mathrm{IBP} 6 \mathrm{~S}}\left(\mathcal{M}_{c}\right)}\right\rceil \times T_{\mathrm{Sym}}+T_{\mathrm{Pre}}+T_{\mathrm{Hdr}}
$$

where $N_{\text {IBP6S }}$ is the number of information bits per six OFDM symbols, which depends on the TM (listed in Table I); $T_{\text {Sym }}$, $T_{\mathrm{Pre}}$, and $T_{\mathrm{Hdr}}$ are the transmission times of one OFDM symbol, the PLCP frame preamble, and the frame header, respectively.

Using the Dly-ACK scheme, the ACK frame piggybacks the link feedback IE, which recommends the adjustment to the data rate and transmission power level. Then, the transmitter may change the TM in the next burst transmission accordingly.

\section{Dly-ACK Scheme and Packet Fragmentation}

In the Dly-ACK scheme, as shown in Fig. 2(a), we call the $B$ data frames plus the acknowledge frame a burst transmission. There is a minimum interframe spacing (MIFS) interval between two consecutive data frames. The last data frame and the ACK frame are separated by a short interframe spacing (SIFS). Given the allocated channel time in the $n$th $\mathrm{RB} \Delta_{n}$, the payload size $L$ of data frames, and the TM $\mathcal{M}_{c}$, the number of frames in a burst, called burst size, is

$$
B_{n, c}(L)=\left\lfloor\frac{\Delta_{n}-T_{\mathrm{ACK}}-2 \times \mathrm{SIFS}-\mathrm{GT}+\mathrm{MIFS}}{T_{F}\left(L, \mathcal{M}_{c}\right)+\mathrm{MIFS}}\right\rfloor
$$

where $T_{\mathrm{ACK}}$ is the transmission time of the ACK frame, GT is the guard time, and $T_{F}\left(L, \mathcal{M}_{c}\right)$ is calculated by (1).

At the transmitting device, each packet may be partitioned into several fragments of equal size, except for the last one. Each fragment is encapsulated in one PLCP frame and delivered using the Dly-ACK scheme. The sequence of the fragments is identified by the sequence number (i.e., the MSDU number) and the fragment number, as illustrated in Fig. 2(b). The receiving device shall reassemble each MSDU in the correct order.

As shown in Fig. 1, each RB contains a Dly-ACK burst transmission. Upon reception of the delayed ACK at the end of an RB, the acknowledged fragments are removed from the buffer. If the vacant space in the buffer is not enough for a packet, the newly arrived packets will be discarded.

For simplicity, we assume that the packet length is fixed as $L_{P}$ bytes and that each packet is partitioned into $M(M \geq 1)$ fragments with the length of $L=L_{P} / M$ bytes. The sender has a queuing buffer of $F$ fragments.

\section{UWB Shadowing Channel Model}

WPANs are usually deployed in office or residential buildings with stationary transceivers. However, obstacles, such as people, may move around the transceivers and obstruct some significant propagation paths. Such body-shadowing effect (BSE) can considerably reduce the received signal power, resulting in channel variation. Measurements of BSE have shown the power attenuation of up to $8 \mathrm{~dB}$ with omni antennas [14], [15]. A packet-level model for the UWB shadowing channel based on a first-order finite-state Markov chain (FSMC) has been proposed in [16] and [17].

We define totally $C$ channel states, and the state $S_{c}(c=$ $1,2, \ldots, C)$ is the received SNR interval for the $c$ th TM, i.e., $\Gamma_{c}$, as mentioned in Section III-B. Because the shadowing effect depends on the location of the body, each channel state corresponds to a spatial zone of the obstructing position. The state transition rates are given by [16]

$$
\begin{cases}\mu_{c, c+1}=\frac{\alpha_{c}}{A_{c} t}, & c=1,2, \ldots, C-1 \\ \mu_{c, c-1}=\frac{1-\alpha_{c}}{A_{c} \bar{t}}, & c=2,3, \ldots, C\end{cases}
$$


where $A_{c}$ is the area of the $c$ th zone, $\bar{t}$ is the average duration for which the person stays in a unit area, and $0 \leq \alpha_{c} \leq 1$ is the probability that the person moves into the adjacent inner zone (state transition from $S_{c}$ to $S_{c+1}$ ). Given the SNR intervals, the average BER of each channel state, which is denoted by $\varepsilon_{c}$, can be obtained according to the MB-OFDM performance [13], [16].

\section{QUeUing ANALYSiS}

We focus on the sender queue, which can be described using a $G / G / B(t) / F$ model. First, the packet arrival is approximated as a Poisson process with the average arrival rate of $\Lambda$ packets/second, and thus, the fragment arrival is a general process (because a packet arrival results in $M$ simultaneous fragment arrivals). Second, the service time of a fragment depends on the number of transmission trials and the arbitrary vacation periods, which has a general distribution. Third, using Dly-ACK, multiple fragments are transmitted in burst and may be removed from the buffer simultaneously. If the maximal burst size is $B(t)$ at time $t$, the system can be viewed as having $B(t)$ servers with vacations, where $B(t)$ is random and depends on the TM and RB durations, as shown in (2). Finally, the sender's buffer size is $F$ fragments.

Because it is very difficult, if not impossible, to apply the traditional queuing analysis, we model the system based on the RSs in the superframes and build a 3-D FSMC, as presented in the following.

\section{A. Markov Model}

A superframe is divided into $N$ RSs from the viewpoint of the tagged node. We define the system state at the beginning of each RS as the triplet of $(n, c, q)$, where $n \in\{1,2, \ldots, N\}$ is the index of the RS, $c \in\{1,2, \ldots, C\}$ is the channel state, and $q \in\{0,1, \ldots, F\}$ is the number of fragments in the buffer. There are $(F+1) N C$ states. The 3-D discrete-time FSMC model can capture the MAC protocol scheduling, channel evolution, and queuing behavior. We use the RSs as the time slots of the model, whose durations are not constant but repeat from $T_{1}$ to $T_{N}$ per scheduling period. The state at the time slot $t$ is denoted by $\left(n_{t}, c_{t}, q_{t}\right)$. The nonnull one-step transition probabilities are derived as follows.

1) Arrival Process: Denote $a_{t}$ as the number of packet arrivals during the $t$ th time slot. Since the queue length is $q_{t}$ fragments at the beginning of the slot, only $\min \left\{a_{t}, A_{t}\right\}$ packets can enter the queue, where $A_{t}=\left\lfloor\left(F-q_{t}\right) / M\right\rfloor$. Denote $b_{t}$ as the number of accommodated fragments, and the probability mass function (PMF) of $b_{t}$ can be obtained as

$$
\begin{array}{r}
f_{b_{t}}\left(a_{t} M \mid n_{t}, q_{t}\right) \\
= \begin{cases}\frac{\left(\Lambda T_{n_{t}}\right)^{a_{t}}}{a_{t} !} e^{-\Lambda T_{n_{t}}}=\Psi\left(a_{t}, \Lambda T_{n_{t}}\right), & a_{t}<A_{t} \\
1-\sum_{x=0}^{A_{t}-1} \Psi\left(x, \Lambda T_{n_{t}}\right), & a_{t}=A_{t} \\
0 & a_{t}>A_{t}\end{cases}
\end{array}
$$

where $\Psi(\cdot)$ is the Poisson distribution function.

2) Channel-State Transition: For the UWB shadowing channel, the residential time in each channel state is much longer than the duration of a time slot $T_{n}(n=1, \ldots, N)$; thus, the probability that the channel state transition occurs more than once in one time slot is negligible. The transition probabilities can be estimated by

$$
\begin{cases}h_{c, c+1}=\frac{\alpha_{c}}{A_{c} t} T_{n_{t}}, & c=1,2, \ldots, C-1 \\ h_{c, c-1}=\frac{\left(1-\alpha_{c}\right)}{A_{c} t} T_{n_{t}}, & c=2,3, \ldots, C \\ h_{c, c}=1-\frac{1}{A_{c} t} T_{n_{t}}, & c=1,2, \ldots, C .\end{cases}
$$

3) Queue Service Process: The maximal burst size in the RB in the th time slot, denoted by $B_{n_{t}, c_{t}}$, can be obtained by (1) and (2). The number of fragments in the burst is $v_{t}=$ $\min \left\{q_{t}+b_{t}, B_{n_{t}, c_{t}}\right\}$. Because the duration of an RB is of several MASs, which is much smaller than the channel coherent time, it is assumed that the channel is static and that the frame error probability is constant within a burst.

By using the AMC mechanism, the PHY layer chooses the appropriate TM to ensure the target BER. However, the TM is determined by $c_{t}$ estimated by the receiver during the previous burst. When the current burst is in transmission using TM $\mathcal{M}_{c_{t}}$, the channel is in state $c_{t+1}$, which can be the same as $c_{t}$ or its adjacent states. If $c_{t+1}=c_{t}$, we have the target BER $\varepsilon_{0}$ and the target frame error rate (FER). If $c_{t+1}=c_{t}+1$, the channel condition becomes worse than expected, and the BER is $\varepsilon_{w}>\varepsilon_{0}$. Similarly, if $c_{t+1}=c_{t}-1$ (the channel condition becomes better), the BER is $\varepsilon_{b}<\varepsilon_{0}$. The FER is $\eta_{c_{t}, c_{t+1}}=$ $1-(1-\varepsilon)^{L}$, where $\varepsilon$ is $\varepsilon_{0}, \varepsilon_{w}$, and $\varepsilon_{b}$ for the three scenarios, respectively. Because the frame header and the ACK frames are sent at the base data rate of $53.3 \mathrm{Mb} / \mathrm{s}$ or lower and protected by strong error-correction coding, we assume that they can correctly be received.

If $d_{t}$ frames are correctly received in a burst transmission during time slot $t$, they will be removed from the buffer. $d_{t}$ is of binomial distribution with PMF of

$$
\begin{aligned}
f_{d_{t}}\left(x \mid v_{t}, c_{t}, c_{t+1}\right) & =\left(\begin{array}{c}
v_{t} \\
x
\end{array}\right)\left(1-\eta_{c_{t}, c_{t+1}}\right)^{x} \eta_{c_{t}, c_{t+1}}^{v_{t}-x} \\
& =\Phi\left(x, v_{t}, 1-\eta_{c_{t}, c_{t+1}}\right)
\end{aligned}
$$

where $\Phi(\cdot)$ is the binomial distribution function.

4) System State-Transition Probabilities: The RS index in the $(t+1)$ th time slot is $n_{t+1}=\left(n_{t} \bmod N\right)+1$, and the queue length at the beginning of the time slot is $q_{t+1}=q_{t}+$ $b_{t}-d_{t}$. The transition probability from state $\left(n_{t}, c_{t}, q_{t}\right)$ to state $\left(n_{t+1}, c_{t+1}, q_{t+1}\right)$ is

$$
\begin{aligned}
& \operatorname{Pr}\left\{\left(n_{t+1}, c_{t+1}, q_{t+1}\right) \mid\left(n_{t}, c_{t}, q_{t}\right)\right\} \\
& \quad=\operatorname{Pr}\left\{c_{t+1} \mid c_{t}, n_{t}\right\} \operatorname{Pr}\left\{b_{t}-d_{t}=q_{t+1}-q_{t} \mid n_{t}, c_{t}, q_{t}, c_{t+1}\right\} .
\end{aligned}
$$

The PMF of the random variable $b_{t}-d_{t}$ can be obtained by

$f_{b_{t}-d_{t}}\left(x \mid n_{t}, c_{t}, q_{t}, c_{t+1}\right)$

$=\sum_{a_{t}=0}^{A_{t}} f_{b_{t}}\left(a_{t} M \mid n_{t}, q_{t}\right) f_{d_{t}}\left(a_{t} M-x \mid b_{t}=a_{t} M, c_{t}, q_{t}, c_{t+1}\right)$

where $f_{b_{t}}$ and $f_{d_{t}}$ are given in (4) and (6), respectively. 
We organize the transition probabilities from state $(n, c, q)$ to all the system states in a column vector as

$$
\begin{aligned}
& \mathbf{P}_{(\mathbf{n}, \mathbf{c}, \mathbf{q})} \\
& =\left[\begin{array}{llll}
\operatorname{Pr}\{(1,1,0) \mid(n, c, q)\} & \cdots & \operatorname{Pr}\{(N, C, F) \mid(n, c, q)\}
\end{array}\right]^{T} .
\end{aligned}
$$

The state transition probability matrix can be obtained as

$\mathbb{P}=\left[\begin{array}{lllllll}\mathbf{P}_{(\mathbf{1}, \mathbf{1}, \mathbf{0})} & \cdots & \mathbf{P}_{(\mathbf{1}, \mathbf{C}, \mathbf{F})} & \cdots & \mathbf{P}_{(\mathbf{N}, \mathbf{1}, \mathbf{0})} & \cdots & \mathbf{P}_{(\mathbf{N}, \mathbf{C}, \mathbf{F})}\end{array}\right]$.

5) Stationary Distribution: Let $\pi_{(n, c, q)}$ be the steadystate probability of $(n, c, q)$, and define the column vector of the steady-state distribution as $\boldsymbol{\Pi}=\left[\pi_{(1,1,0)} \quad \ldots\right.$ $\left.\pi_{(1, C, F)} \quad \cdots \quad \pi_{(N, 1,0)} \quad \cdots \quad \pi_{(N, C, F)}\right]^{T}$, which can be obtained by solving the linear equations of

$$
\left\{\begin{array}{l}
\boldsymbol{\Pi}=\mathbb{P} \boldsymbol{\Pi} \\
\sum_{n=1}^{N} \sum_{c=1}^{C} \sum_{q=0}^{F} \pi_{(n, c, q)}=1 .
\end{array}\right.
$$

Denote $Q$ as the queue length at the beginning of an RS. The stationary distribution of $Q$ is $f_{Q}(q)=\sum_{n=1}^{N} \sum_{c=1}^{C} \pi_{(n, c, q)}$.

\section{B. $P D R$}

Considering that AMC in the PHY layer can bound the $\mathrm{BER}$, the probability that a frame is discarded due to excessive retransmissions is negligible. Because of the arbitrary length of vacation time, we evaluate the buffer overflow probability and PDR for each RS.

Denote the queue length at the beginning of the $n$th RS in the superframe as $Q_{n}$ and the stationary distribution of $Q_{n}$ is

$$
f_{Q_{n}}(q)=\sum_{c=1}^{C} \pi_{n, c, q}
$$

Let $A_{n}$ denote the maximal number of packets accommodated in the $n$th slot. The PMF of $A_{n}$ is $f_{A_{n}}(x)=$ $\sum_{q=F-(x+1) M+1}^{F-x M} f_{Q_{n}}(q), x=0,1, \ldots,\lfloor F / M\rfloor$.

The number of dropped packets during the $n$th $\mathrm{RS}$ is denoted by $D_{n}$, and thus, $a_{n}=A_{n}+D_{n}$ is the total number of packets arriving during the slot. The conditional probability of $D_{n}$ is $f_{D_{n}}\left(x \mid A_{n}=y\right)=f_{a_{n}}(x+y)=\Psi\left(x+y, \Lambda T_{n}\right)$. Thus, the average number of dropped packets in the $n$th $\mathrm{RS}$ is

$$
\begin{aligned}
\bar{D}_{n} & =\sum_{y=0}^{\left\lfloor\frac{F}{M}\right\rfloor} \sum_{x=1}^{\infty} x f_{D_{n}}\left(x \mid A_{n}=y\right) f_{A_{n}}(y) \\
& =\sum_{y=0}^{\left\lfloor\frac{F}{M}\right\rfloor} \sum_{x=1}^{\infty}\left[x \Psi\left(x+y, \Lambda T_{n}\right) \sum_{q=F-(y+1) M+1}^{F-y M} f_{Q_{n}}(q)\right] .
\end{aligned}
$$

Given the average number of packets dropped in one superframe, the PDR is $\bar{D}=\sum_{n=1}^{N} \bar{D}_{n} /\left(\Lambda T_{\mathrm{SF}}\right)$.

\section{Queuing Delay}

The total delay consists of the queuing delay and the transmission delay. The queuing delay of a fragmented packet is defined as the duration from the packet arrival instant until the beginning of the earliest RB in which the first fragment of the packet is transmitted. The transmission delay is the time interval from the transmission of the first fragment until all the fragments of the packet are correctly received. In this section, we derive the queuing delay distribution. The transmission delay will be discussed in the next section.

The queuing delay of a packet contains two parts: 1) the delay to the end of the RS in which the packet arrives and 2) the delay to the RB where the first fragment is transmitted. For the system state $(n, c, q)$, the buffer can accommodate, at most, $A_{n, q}=\lfloor(F-q) / M\rfloor$ new packets. The average number of the accommodated packets is

$$
\bar{a}_{n, q}=\sum_{x=0}^{A_{n, q}-1} x \Psi\left(x, \Lambda T_{n}\right)+A_{n, q}\left[1-\sum_{x=0}^{A_{n, q}-1} \Psi\left(x, \Lambda T_{n}\right)\right] .
$$

Because the average arrival interval is $\delta=1 / \Lambda$, the expected arriving instant of the $i$ th $\left(i=1,2, \ldots, \bar{a}_{n, q}\right)$ packet is $i \delta$ with respect to the beginning of the RS.

The number of fragments in the buffer when the $i$ th packet arrives is $Q_{i}=q+(i-1) M$. Let $G_{i}$ denote the expected number of RSs the $i$ th packet experiences while it is waiting in the buffer before its first fragment is transmitted. $G_{i}$ is

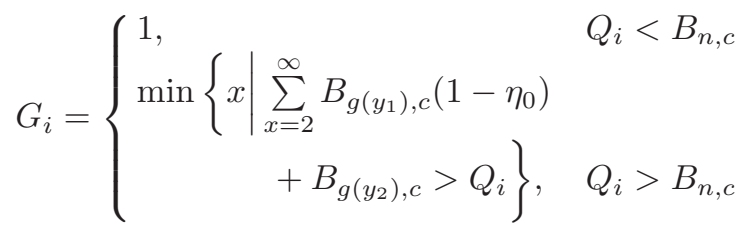

where $y_{1}=n+x-2, y_{2}=n+x-1$, and $g(y)=[(y-$ 1) $\bmod N]+1 . B_{g(y), c}$ is the burst size of the $g(y)$ th RB using $\mathrm{TM} \mathcal{M}_{c}$, as given in (2).

Thus, the total queuing delay for the $i$ th packet is

$$
\zeta_{i}=\sum_{j=n}^{n+G_{i}-1} T_{g(j)}-i \delta-\Delta_{g\left(n+G_{i}-1\right)} .
$$

The average queuing delay of the packets arriving during the system state $(n, c, q)$ is $\bar{\zeta}_{(n, c, q)}=\left(1 / \bar{a}_{n, q}\right) \sum_{i=1}^{\bar{a}_{n, q}} \zeta_{i}$. Thus, the average queuing delay for all the system states is

$$
\begin{aligned}
\bar{\tau}_{q} & =\sum_{n=1}^{N} \sum_{c=1}^{C} \sum_{q=0}^{F-M} \bar{\zeta}_{(n, c, q)} \pi_{(n, c, q)} \\
& =\sum_{n=1}^{N} \sum_{c=1}^{C} \sum_{q=0}^{F-M}\left(\frac{1}{\bar{a}_{n, q}} \sum_{i=1}^{\bar{a}_{n, q}} \zeta_{i}\right) \pi_{(n, c, q)} .
\end{aligned}
$$

\section{Transmission Delay of Fragmented PaCKets}

To quantify the transmission delay of a fragmented packet, we first obtain the PMF of the number of burst transmissions 
needed for a packet, and the transmission delay is acquired by considering the reservation pattern in the superframe.

\section{A. Transmission Process of a Fragmented Packet}

A fragment is called imported in a burst when it is transmitted for the first time. We index the burst in which the first fragment of the tagged packet is imported as the first burst, and the following burst as the second one, and so on. Suppose that the last fragment of the tagged packet is imported in the $U$ th burst. The number of fragments imported in the $u$ th $(u=$ $1,2, \ldots, U)$ burst is denoted by $m_{u}$. The vector of $\mathbf{m}_{\mathbf{U}}=$ $\left[\begin{array}{llll}m_{1} & m_{2} & \cdots & m_{U}\end{array}\right]$ is called import vector, and we have $\sum_{u=1}^{U} m_{u}=M$.

\section{B. PMF of the Number of Bursts for One Packet}

With slow fading, the probability for the channel to change state during the transmission of one packet is much less than the probability to stay in the same state. Therefore, due to the space limit, we assume that the channel is constant during the transmission of the tagged packet, and thus, the FER is $\eta_{0}$, as defined earlier. The analysis of the scenario that the channel state changes can be found in [18].

Let $W_{u}^{\prime}$ denote the index of the burst where one of the $m_{u}$ fragments, which is imported in the $u$ th burst, is delivered. The PMF and cumulative distribution function (cdf) of $W_{u}^{\prime}$, respectively, are $(w \geq u)$

$$
\begin{aligned}
f_{W_{u}^{\prime}}(w) & =\left(1-\eta_{0}\right) \eta_{0}^{w-u} \\
F_{W_{u}^{\prime}}(w) & =\sum_{x=u}^{w}\left(1-\eta_{0}\right) \eta_{0}^{x-u}=1-\eta_{0}^{w-u+1} .
\end{aligned}
$$

Note that $W_{u}^{\prime}$ for each of the $m_{u}$ fragments are independent and identically distributed random variables. Denote $W_{u}$ as the burst when all the $m_{u}$ fragments are received. The cdf of $W_{u}$ is $(w \geq u)$

$$
F_{W_{u}}\left(w \mid m_{u}\right)=\left[F_{W_{u}^{\prime}}(w)\right]^{m_{u}}=\left(1-\eta_{0}^{w-u+1}\right)^{m_{u}} .
$$

Denote $W$ as the last burst when all the $M$ fragments have been delivered. Given the import vector $\mathbf{m}_{\mathbf{U}}$ (the import process includes $U$ bursts), the conditional cdf of $W$ is $(w \geq U)$

$$
F_{W}\left(w \mid \mathbf{m}_{\mathbf{U}}\right)=\prod_{u=1}^{U} F_{W_{u}}\left(w \mid m_{u}\right)=\prod_{u=1}^{U}\left(1-\eta_{0}^{w-u+1}\right)^{m_{u}} .
$$

Finally, the unconditional cdf of $W$, i.e., the probability that the tagged packet can be delivered within $w$ bursts, is

$$
F_{W}(w)=\sum_{U=1}^{w} F_{W}\left(w \mid \mathbf{m}_{\mathbf{U}}\right) \operatorname{Pr}\left\{\mathbf{m}_{\mathbf{U}}\right\}
$$

where $\operatorname{Pr}\left\{\mathbf{m}_{\mathbf{U}}\right\}$ is the probability of the import vector $\mathbf{m}_{\mathbf{U}}$ (will be discussed in the next section). Then, the PMF of $W$ can be obtained by $f_{W}(w)=F_{W}(w)-F_{W}(w-1)$.

\section{Import Process}

Because the import process is related to the burst size, we evaluate $\operatorname{Pr}\left\{\mathbf{m}_{\mathbf{U}}\right\}$ for each RB. Suppose that the tagged packet is first imported in the $n$th $\mathrm{RB}$. At the beginning of the $\mathrm{RB}$, the number of fragments in the buffer is $Q_{n}^{\prime}=Q_{n}+b_{n}$, where $Q_{n}$ is the queue length at the beginning of the RS as defined earlier, and $b_{n}$ is the fragment arrivals during the RS. If the TM is $\mathcal{M}_{c}$ and $Q_{n}^{\prime}>B_{n, c}$, the burst transmission in the RB will be full, and there is at least one fragment in the buffer that is not transmitted. We call such scenario as being saturated.

1) For $U=1$ : We first consider the saturated case. Here, we make two approximations. First, because the current burst is saturated, we assume that the previous burst is full (which happens with high probability). Second, the number of the remaining fragments of the previous packet that have not yet been imported is uniformly distributed over $[1, M-1]$. As shown in the numerical results, these approximations do not affect the accuracy of the analytical results.

Thus, the number of the fragments of new packets that can be imported in the burst is

$$
V=\left[B_{n, c}-B_{g(n-1), c}\left(1-\eta_{0}\right)-\frac{M-1}{2}\right]
$$

where $[\cdot]$ rounds the value inside to the nearest integer. Since the number of packets completely imported is $\lfloor V / M\rfloor$ and the last packet is partially imported, the probability for one packet to be completely imported $(U=1)$ is

$$
\operatorname{Pr}\left\{U=1 \mid Q_{n}^{\prime}>B_{n, c}\right\}=\frac{\left\lfloor\frac{V}{M}\right\rfloor}{\left\lfloor\frac{V}{M}\right\rfloor+1} .
$$

Second, for the unsaturated scenario, because all packets in the buffer can be completely imported, we have $\operatorname{Pr}\{U=$ $\left.1 \mid Q_{n}^{\prime} \leq B_{n, c}\right\}=1$.

The PMF of $Q_{n}^{\prime}$ can be obtained by

$$
f_{Q_{n}^{\prime}}(q)=\sum_{x=0}^{q} f_{Q_{n}}(x) f_{b_{n}}(q-x \mid n, x)
$$

where $f_{Q_{n}}(x)$ and $f_{b_{n}}(x)$ are given in (12) and (4), respectively. Thus, the probability for the $n$th RB to be saturated is $\operatorname{Pr}\left\{Q_{n}^{\prime}>B_{n, c}\right\}=\sum_{q=B_{n, c}+1}^{F} f_{Q_{n}^{\prime}}(q)$.

Obviously, when $U=1$, the packet is completely imported in one burst, and the import vector is simply $\mathbf{m}_{\mathbf{U}}=[M]$. The probability is

$$
\begin{aligned}
\operatorname{Pr} & \left\{\mathbf{m}_{\mathbf{U}}=[M] \mid n, c\right\} \\
= & \operatorname{Pr}\left\{U=1 \mid Q_{n}^{\prime}>B_{n, c}\right\} \operatorname{Pr}\left\{Q_{n}^{\prime}>B_{n, c}\right\} \\
& +\operatorname{Pr}\left\{U=1 \mid Q_{n}^{\prime} \leq B_{n, c}\right\} \operatorname{Pr}\left\{Q_{n}^{\prime} \leq B_{n, c}\right\} \\
& =1-\left(1-\frac{\left\lfloor\frac{V}{M}\right\rfloor}{\left\lfloor\frac{V}{M}\right\rfloor+1}\right) \sum_{q=B_{n, c}+1}^{F} f_{Q_{n}^{\prime}}(q) .
\end{aligned}
$$

2) For $U>1$ : The import vector is $\mathbf{m}_{\mathbf{U}}=$ $\left[\begin{array}{llll}m_{1} & m_{2} & \cdots & m_{U}\end{array}\right]$, where $m_{u} \in\{1,2, \ldots, M-1\}$ for $u=1$ or $U$, and $m_{u} \in\{0,1, \ldots, M-2\}$ for $1<u<U$. First, $m_{1}$ is approximated to be uniformly distributed, and 
the PMF is $f_{m_{1}}=1 /(M-1)$. Then, $m_{u}$ fragments being imported in the $u$ th $(1<u<U)$ burst means that exactly $m_{u}$ fragments are successfully delivered in the previous burst, which is in the $g(n+u-2)$ th RB. Therefore, the PMF of $m_{u}$ can be obtained as $f_{m_{u}}(x \mid n, c)=\Phi\left(x, B_{g(n+u-2), c}, 1-\eta_{0}\right)$, where $0 \leq x \leq M-2$. Finally, the probability for the last $m_{U}$ fragments to be imported in the $U$ th burst (thus, the import process includes $U$ bursts) is $f_{m_{U}}(x \mid n, c)=$ $\sum_{y=x}^{B_{g(n+U-2), c}} \Phi\left(y, B_{g(n+U-2), c}, 1-\eta_{0}\right), \quad$ where $\quad 1 \leq x \leq$ $M-1$.

Thus, the probability of the import vector given that the packet's first fragment is imported in the $n$th $\mathrm{RB}$ and the TM is $\mathcal{M}_{c}$ is

$$
\begin{aligned}
& \operatorname{Pr}\left\{\mathbf{m}_{\mathbf{U}}=\left[\begin{array}{llll}
m_{1} & m_{2} & \cdots & m_{U}
\end{array}\right] \mid n, c\right\} \\
& =\operatorname{Pr}\{U>1\} f_{m_{1}}\left(m_{1}\right) f_{m_{2}}\left(m_{2} \mid n, c\right) \cdots f_{m_{U}}\left(m_{U} \mid n, c\right) \\
& =(1-\operatorname{Pr}\{U=1\}) \frac{1}{M-1} \\
& \times\left[\prod_{u=2}^{U-1} \Phi\left(m_{u}, B_{g(n+u-2), c}, 1-\eta_{0}\right)\right] \\
& B_{g(n+U-2), c} \\
& \times \quad \sum_{y=x}^{B_{g(n+U-2), c}} \Phi\left(y, B_{g(n+U-2), c}, 1-\eta_{0}\right) .
\end{aligned}
$$

Plugging (26) and (27) into (22), $F_{W}(w \mid n, c)$ can be obtained.

\section{Transmission Delay}

If all $M$ fragments are delivered in $w$ bursts, the transmission delay is $\Delta_{n}+\sum_{j=n+1}^{n+w-1} T_{g(j)}$. The average transmission delay for a packet that is first time imported in the $n$th RB is

$$
\bar{\xi}_{n, c}=\sum_{w=1}^{\infty} f_{W}(w \mid n, c)\left(\Delta_{n}+\sum_{j=n+1}^{n+w-1} T_{j}\right) .
$$

To estimate the average transmission delay using $\mathrm{TM} \mathcal{M}_{c}$, we need to determine the probability for one packet to be first time imported in the $n$th RB, which is denoted by $p_{n, c}$. We propose Algorithm 1 to estimate $p_{n, c}$.

Algorithm 1 Throughput estimation of each RB

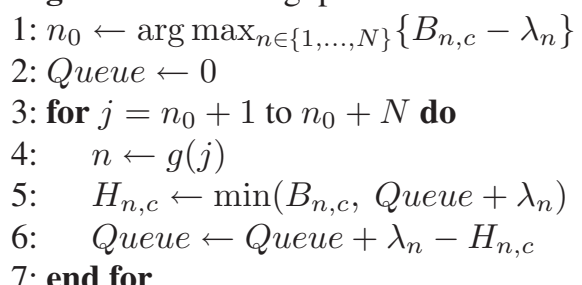

Then, the probability for one packet to be first imported in the $n$th RB is $p_{n, c}=H_{n, c} / \sum_{n=1}^{N} H_{n, c}$. Finally, the average transmission delay of a packet using TM $\mathcal{M}_{c}$ (i.e., in the channel state $S_{c}$ ) is

$$
\bar{\xi}_{c}=\sum_{n=1}^{N} p_{n, c} \bar{\xi}_{n, c}
$$

\section{JoInt ERror-Control Mechanism Optimization}

In Sections IV and V, we have studied the performance of the joint error-control mechanism. In [8], an optimal ACK scheme was presented by adapting the payload size only. However, how to optimize the TM (i.e., the SNR boundaries), the target BER, and the fragment size to achieve the best performance is an open issue. Here, we define the optimization problem as maximizing the link throughput via jointly arranging the TM in the PHY layer and the fragment size in the link layer.

\section{A. Throughput Optimization Problem}

Since the BER depends on both the received SNR $\gamma$ and the TM, it is denoted by $\varepsilon\left(\gamma, \mathcal{M}_{c}\right)$. Given the channel time allocated to the tagged user, i.e., $\Delta_{n}(n=1,2, \ldots, N)$, the link throughput of the saturated sender can be expressed as

$$
H\left(\gamma, \mathcal{M}_{c}, L\right)=\frac{8 L}{T_{\mathrm{SF}}}\left(\sum_{n=1}^{N} B_{n, c}\right)\left[1-\varepsilon\left(\gamma, \mathcal{M}_{c}\right)\right]^{8 L}
$$

where $B_{n, c}$ is given by (2). To maximize $H\left(\gamma, \mathcal{M}_{c}, L\right)$ given $\gamma$, the two-step numerical search for the optimal system parameters is as follows.

First, we determine the optimal fragment size of each TM for fixed SNR. Smaller fragment size leads to higher percentage of the overheads (the fixed frame header, interframe spacing time, and $\mathrm{ACK}$ ) and reduces channel utilization. However, with an error-prone channel condition, a larger frame is more likely to be corrupted, and the link throughput may be reduced.

We can approximate the transmission time of a data frame in (1) by

$$
T_{F}^{\prime} \approx \frac{48 L T_{\mathrm{Sym}}}{N_{\mathrm{IBP} 6 \mathrm{~S}}}+T_{\mathrm{Pre}}+T_{\mathrm{Hdr}} .
$$

By plugging (31) and (2) (ignoring the flooring function) into (30), we can get the throughput as

$$
H^{\prime} \approx \frac{X L\left[1-\varepsilon\left(\gamma, \mathcal{M}_{c}\right)\right]^{8 L}}{L+Y}
$$

where

$$
\begin{aligned}
& X=\frac{N_{\mathrm{IBP} 6 \mathrm{~S}}}{6 T_{\mathrm{Sym}} T_{\mathrm{SF}}} \sum_{n=1}^{N}\left(\Delta_{n}-T_{\mathrm{ACK}}-2 \mathrm{SIFS}+\mathrm{MIFS}\right) \\
& Y=\frac{N_{\mathrm{IBP} 6 \mathrm{~S}}}{48 T_{\mathrm{Sym}}}\left(T_{\mathrm{Pre}}+T_{\mathrm{Hdr}}+\mathrm{MIFS}\right) .
\end{aligned}
$$

Taking the first derivative of (32) and setting it to zero, the approximate optimal fragment length can be obtained as

$L_{c}^{*}(\gamma) \approx \frac{-8 Y \ln (1-\varepsilon)-\sqrt{[8 Y \ln (1-\varepsilon)]^{2}-32 Y \ln (1-\varepsilon)}}{16 \ln (1-\varepsilon)}$.

Second, we can determine the optimal TM for any given SNR $\gamma$ by $c^{*}=\arg \max _{c \in\{1, \ldots, C\}}\left\{H\left[\gamma, \mathcal{M}_{c}, L_{c}^{*}(\gamma)\right]\right\}$; thus, the TM $\mathcal{M}_{c^{*}}$ (with the corresponding optimal fragment size) 
has the maximal throughput. Finally, the SNR interval $\Gamma_{c}$ can be obtained as the SNR range, where $\mathcal{M}_{c}$ is the optimal TM.

\section{B. Suboptimal Joint Error-Control Mechanism}

According to the numerical results about Section VI-A, for a fixed TM, the optimal payload size significantly varies with the SNR of $\gamma \in\left[\gamma_{C}, \gamma_{0}\right]$, which introduces considerable implementation complexity because the system keeps changing the fragment size with the time-varying SNR. However, once AMC is adopted and the appropriate TM is selected, as long as the fragment size is chosen within the appropriate range for the SNR interval of $\Gamma_{c}$ (e.g., from 370 bytes to 2730 bytes for $\mathrm{TM} \mathcal{M}_{3}$ as listed in Table I), the throughput remains almost the same.

This observation suggests using a fixed fragment length to approximate the optimal fragment lengths if AMC is employed in the PHY layer, which can greatly simplify the implementation of fragmentation in practical systems. Considering the ranges of the optimal payload size for each TM listed in Table I, we select the fixed fragment size of $L^{*}=1000$ bytes. For simplicity, we call the error-control mechanism using the fixed $L^{*}$ and optimal TM as the suboptimal strategy. As shown in the numerical results, the performance of this suboptimal strategy is quite close to that of the optimal strategy. In addition, the performance analytical model proposed in Sections IV and $\mathrm{V}$ is directly applicable for this suboptimal error-control mechanism.

\section{ViI. Performance Evaluation}

We simulate a typical WPAN deployed in an indoor environment. The received SNR varies in the range of $[1,7] \mathrm{dB}$ due to the random shadowing. The four TMs of 53.3, 80, 106.7, and $160 \mathrm{Mb} / \mathrm{s}$ that can operate in this range [2] are considered. ${ }^{2}$ The packet size is $4 \mathrm{kB}$ (the maximal allowable payload size of a PLCP frame). Using the parameters of ECMA-368 [2], we first calculate the saturation throughput of different errorcontrol mechanisms and obtain the optimal SNR partition and BERs of AMC. Then, the queuing and transmission processes are simulated and compared.

\section{A. Optimal TM and Fragment Size}

The optimal TM and fragment size are calculated according to Section VI-A and listed in Table I. For example, when the SNR is in the range of $[6.15,7) \mathrm{dB}$, the best $\mathrm{TM}$ is $160 \mathrm{Mb} / \mathrm{s}$, and the optimal payload size varies from 820 bytes (when the SNR is $6.15 \mathrm{~dB}$ ) to 2620 bytes (when the SNR is $7 \mathrm{~dB}$ ). To compare the performance gain, Fig. 3 shows the throughput of five adaptation strategies: 1) the optimal strategy; 2) the TM-adaptation strategy using the optimal TM without fragmentation $(M=1) ; 3)$ the fragment-adaption strategy using the optimal fragment size and fixed TM $(106.7 \mathrm{Mb} / \mathrm{s})$; 4) the suboptimal joint error control designed in Section VI (optimal

${ }^{2}$ If the received SNR is larger, the TMs with higher data rates $(200,320,400$, and $480 \mathrm{Mb} / \mathrm{s}$ [2]) can be used. The analytical model is still applicable.

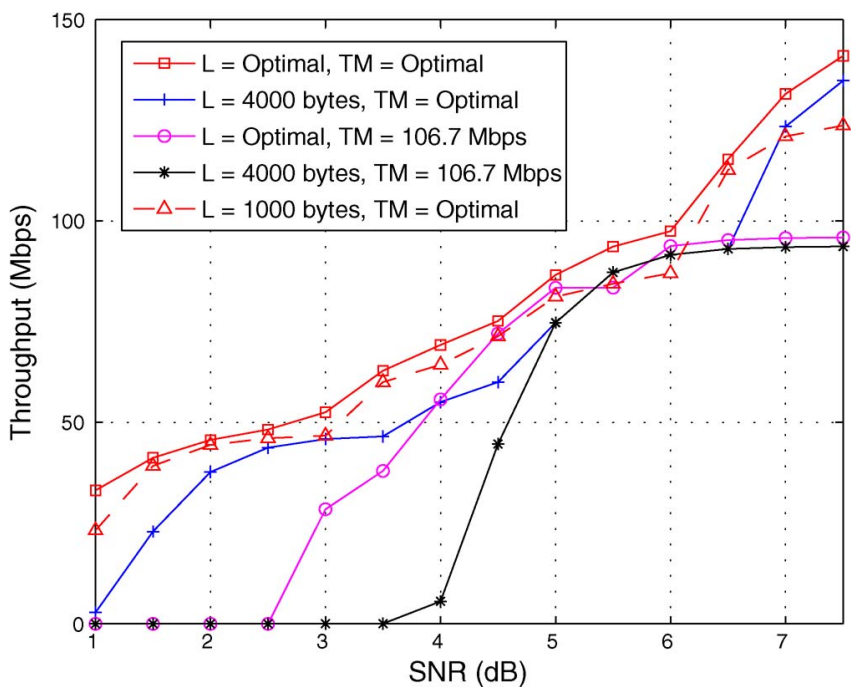

Fig. 3. Throughput of different error-control strategies.

TM with the fixed fragment size of 1000 bytes), which is referred to as joint adaptation; and 5) nonadaptive strategy using $106.7 \mathrm{Mb} / \mathrm{s}$ and no fragmentation. We have the following observations.

First, the joint error control by combining AMC and fragmentation can significantly increase the throughput, particularly for the low-SNR range. For example, when $\gamma=3.5 \mathrm{~dB}$, the optimal strategy is $\mathcal{M}_{c}^{*}=80 \mathrm{Mb} / \mathrm{s}$ and $L^{*}=930$ bytes, and it can achieve around $64 \mathrm{Mb} / \mathrm{s}$ throughput. However, the throughput is almost zero for the nonadaptive strategy and $35 \mathrm{Mb} / \mathrm{s}$ only for the fragment-adaptation strategy (the scenario studied in [8]).

Second, as discussed in Section VI, the suboptimal jointadaptation strategy can achieve the close-to-optimal performance but is much simpler to implement because frequently changing fragment size is avoided.

Third, as shown in Table I, the average BERs of different TMs within their corresponding SNR intervals are in the same order. Therefore, we can approximate the target BER of AMC by the average BER of all TMs, which are denoted by $\varepsilon_{0}=9.59 \times 10^{-6}$. Furthermore, if the TM $\mathcal{M}_{c}(c=1,2,3)$ is used but the channel changes to the adjacent state $S_{c+1}$ (as mentioned in Section IV-A), the BER $\varepsilon_{w}$ of each $\mathcal{M}_{c}$ is closed to one another and can be approximated by $\varepsilon_{w}=2.55 \times 10^{-4}$. Similarly, when $\mathcal{M}_{c}(c=2,3,4)$ is used but the channel changes to $S_{c-1}$, the BERs can be approximated by $\varepsilon_{b}=$ $2.62 \times 10^{-7}$.

\section{B. Queuing and Transmission Simulations}

1) Simulation Settings: Using the SNR ranges of the TMs in Table I as the boundaries of the channel states, the obstructing zones are obtained according to [16]. Assuming that $\alpha_{c}=1 / 2(c=1, \ldots, 4)$, the state transition rates of the FSMC channel model are $\mu_{1,2}=0.20, \mu_{2,3}=\mu_{2,1}=0.11, \mu_{3,4}=$ $\mu_{3,2}=1.02$, and $\mu_{4,3}=3.82$. Then, the transition probabilities of the channel states during each RS can be obtained from (5). In the link layer, the tagged user is allocated two RBs per superframe and eight MASs in each block, which are located 


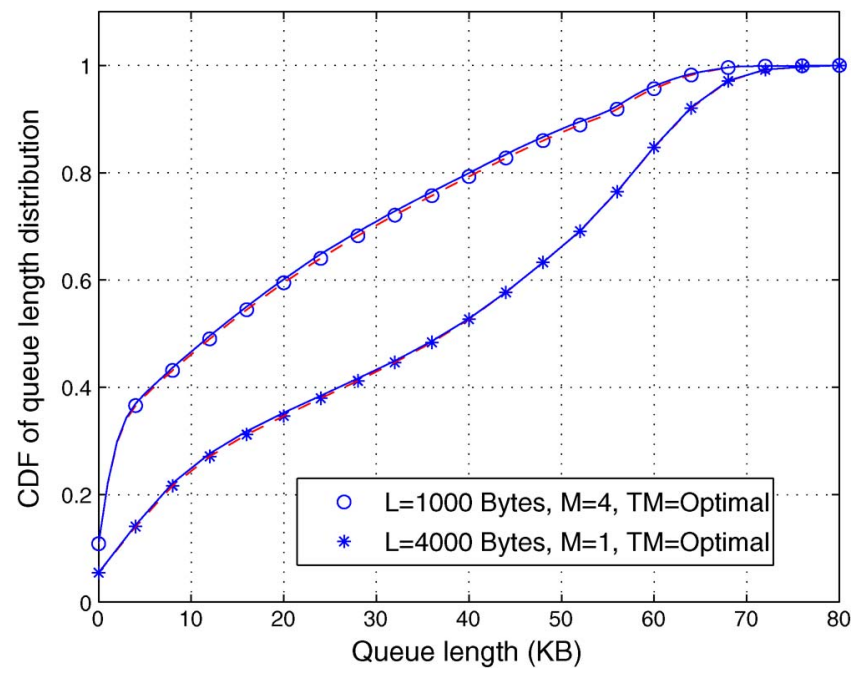

Fig. 4. Stationary distribution (cdf) of queue length.

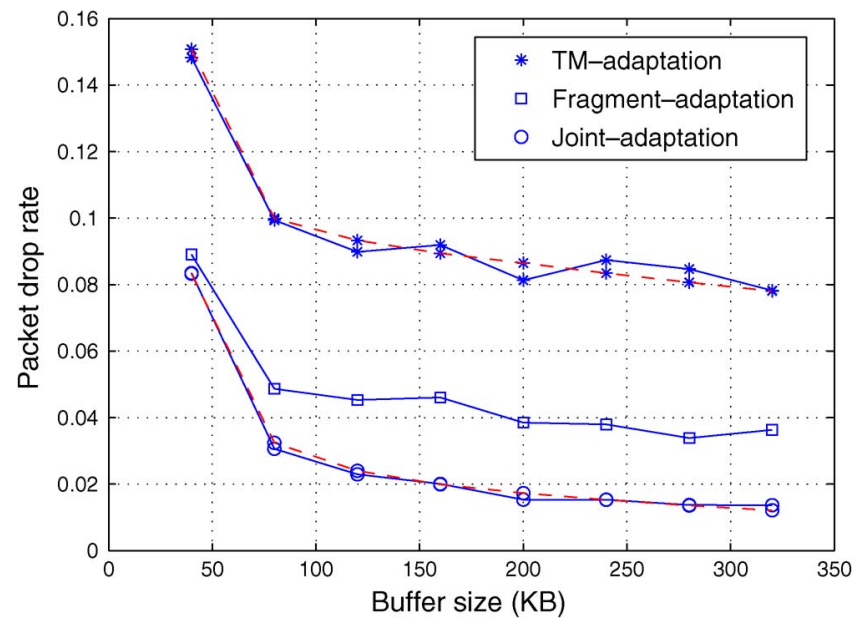

Fig. 5. PDR of the three error-control mechanisms.

at $129-136$ and 193-200 (the numbers denote the MAS index $\in\{1,2, \ldots, 256\})$.

The traffic load is $4.8 \mathrm{Mbps}$, and thus, the packet arrival rate (with packet size of $4 \mathrm{kB}$ ) is $\Lambda=600$ packets/s. When the payload size is $4 \mathrm{kB}$ (no fragmentation), the burst sizes of Dly-ACK in each RB are nine, six, four, and three frames for the four TMs, respectively. When the payload size is 1000 bytes, the burst sizes are 32, 22, 17, and 12 frames, respectively. In Figs. 4-9, the dashed lines represent our analytical results, whereas the solid lines represent the simulation results.

2) Simulation Results: Fig. 4 shows the cdf of the stationary queue length distribution with the buffer size of $80 \mathrm{kB}$. The good agreement between the analytical and simulation results validates the accuracy of our analysis. We can see that fragmentation makes a significant difference in the queue length distribution. With fragmentation $(M=4)$, the queue length has much higher probability to have small values (e.g., smaller than $20 \mathrm{kB}$ with probability of $60 \%$ ), whereas without fragmentation, the queue length has large dynamics (e.g., smaller than $20 \mathrm{kB}$ with probability of $35 \%$ ). This is because the link throughput is increased by using fragmentation, and more importantly, a packet can be partially delivered. Thus, fragmentation can

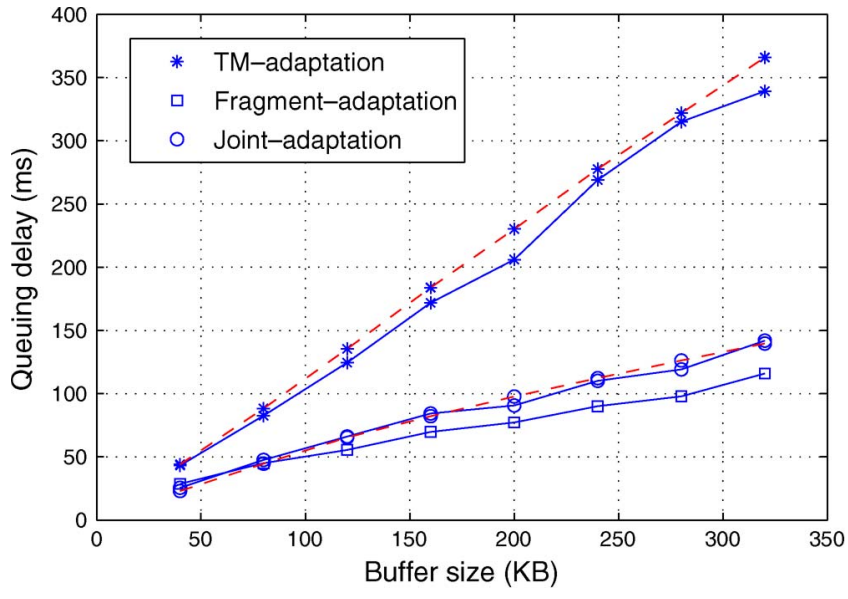

Fig. 6. Queuing delay of the three error-control mechanisms.

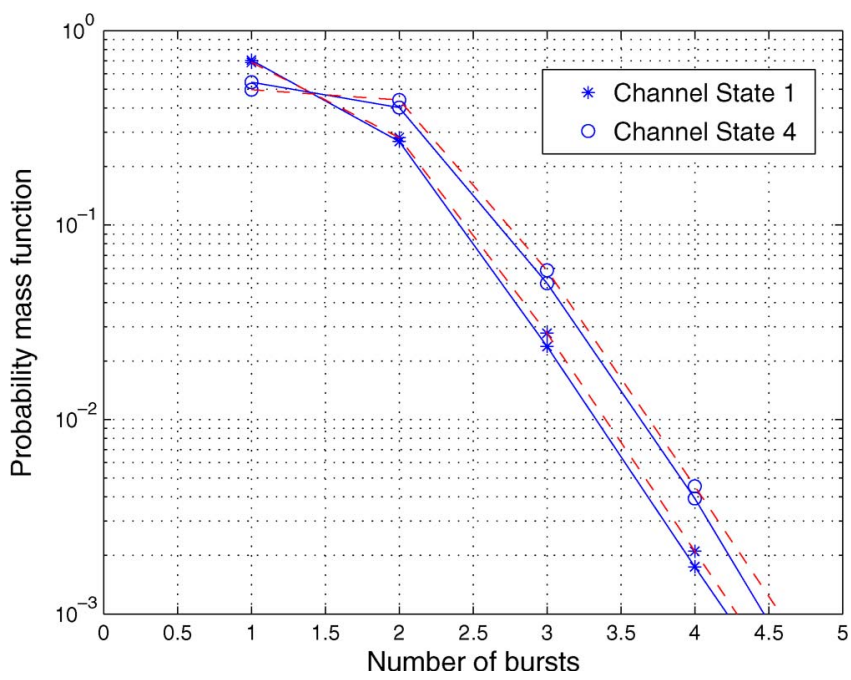

Fig. 7. PMF of $W$.

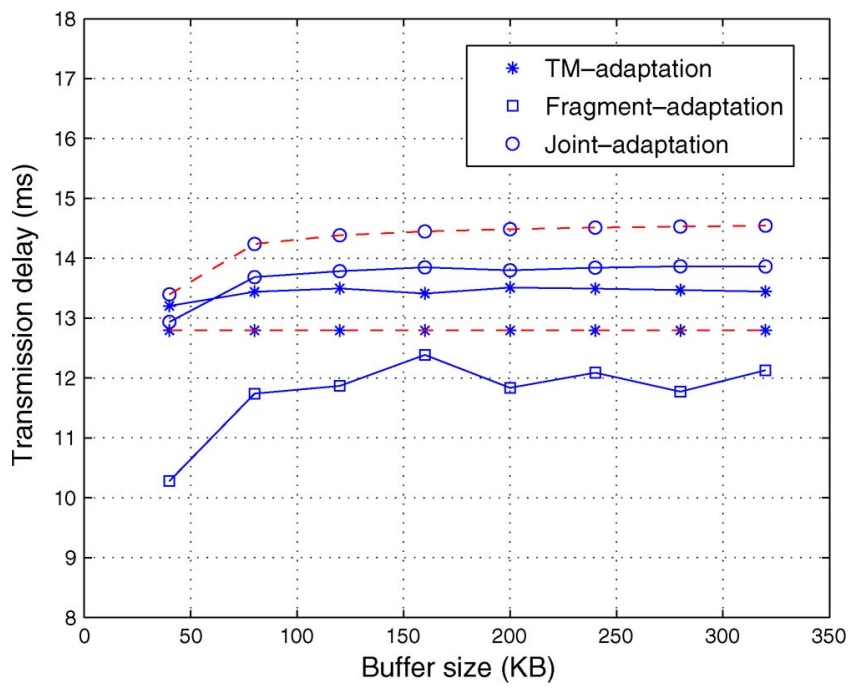

Fig. 8. Transmission delay of the three error-control mechanisms.

improve the queuing behavior of the buffer and thus helps accommodate bursty traffic.

Fig. 5 shows the PDR of three error-control strategies. The fragment-adaptation strategy has lower PDRs than the 


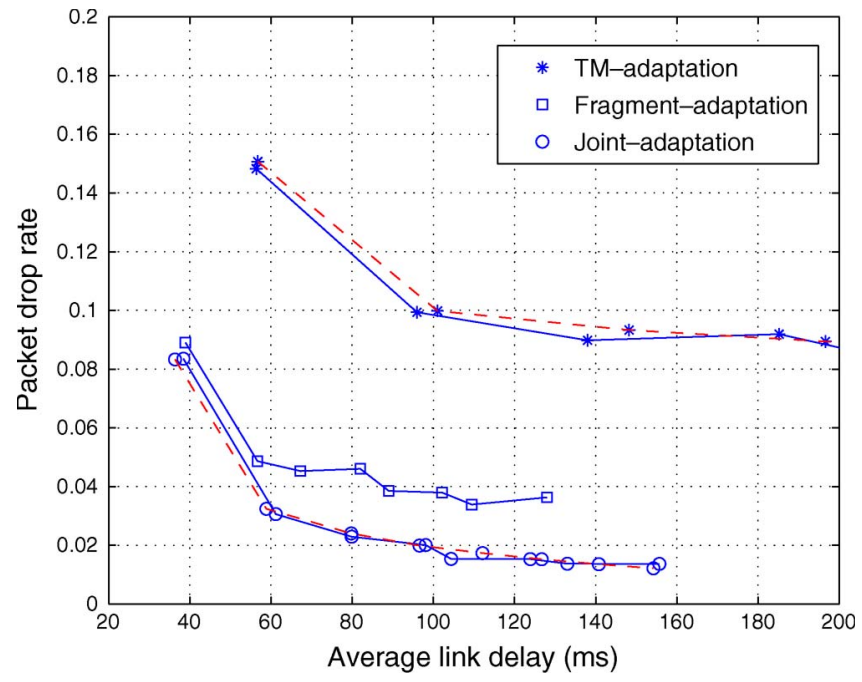

Fig. 9. System performance of the three error-control mechanisms.

TM-adaptation strategy. Although both of them can increase channel utilization by reducing the FER, the fragmentadaptation strategy can improve the queue length distribution and thus further reduce the probability of buffer overflow. However, the PDR of the fragment-adaptation strategy is still much higher than that of the joint-adaptation strategy when the channel condition is bad (such as in channel state $S_{4}$ ); thus, the joint-adaptation mechanism has the lowest average PDR.

Fig. 6 shows the average queuing delay, where the fragmentadaptation strategy has the best performance. Note that the payload size of the fragment-adaptation strategy is always the optimal one, whereas it is fixed as $L=1000$ bytes for the jointadaptation strategy (the suboptimal strategy). Therefore, the queuing delay of the joint-adaptation strategy is slightly larger.

In the following, we investigate the transmission delay. To validate our derivation, Fig. 7 shows $f_{W}(w)$ of a fragmented packet $(M=4)$ for channel states $S_{1}$ and $S_{4}$. The analytical and simulation results match quite well. Most packets can be delivered in two bursts. In the bad state $S_{4}$, the probability of more bursts to deliver a packet is increased, as expected.

The average transmission delays are compared in Fig. 8. With fragmentation, the fragments of one packet are usually imported through several bursts and thus require more bursts to deliver. Therefore, the joint-adaptation strategy has larger transmission delay than the TM-adaptation strategy. However, the fragmentadaptation strategy can provide the smallest transmission delay. This is because when the channel state is good (such as $S_{1}$ ), the packets are not fragmented, and the transmission delay is similar to that of the TM-adaptation strategy. When the channel state is bad (such as $S_{4}$ ), the fragment-adaptation strategy can use smaller fragment size to reduce the FER and retransmissions. In addition, the burst size is increased, and the probability of importing the whole packet in one burst $(U=1)$ is still high.

Another point is that, if the packets are fragmented, the transmission delay is also related to the queuing behavior. As shown in Fig. 8, the transmission delay of the TM-adaptation strategy (no fragmentation) is constant. However, for the payload- and joint-adaptation strategies, the transmission delay is smaller with the small buffer size (such as $40 \mathrm{kB}$ ). This is because, with a smaller buffer, all fragments of one packet are more likely to be imported in less bursts due to the smaller queue length (e.g., the probability of $U=1$ is higher). Thus, the transmission delay is reduced. Finally, there is a gap between the analytical and simulation results. This is because it is difficult to get the accurate probability for one packet to be first imported in the $n$th RB. Instead, we use Algorithm 1 to obtain an estimation of $p_{n, c}$.

Finally, Fig. 9 shows the system performance, i.e., PDR versus average link delay (queuing delay plus transmission delay). As mentioned earlier, the fragment-adaptation strategy has the smallest queuing and transmission delay by using the optimal payload size. However, given the link delay, the jointadaptation strategy provides the lowest PDR. This is because the bandwidth utilization of the fragment-adaptation strategy is much lower than that of the joint-adaptation strategy in bad channel states, which results in much higher PDR. Because the advantages of joint adaptation are more significant under bad channel conditions, it has the best overall performance over the fading channel.

\section{CONCLUSION}

We have comprehensively studied the performance of errorcontrol mechanisms and reservation-based MAC for wireless networks over fading channels. A general queuing model and a suboptimal joint-adaptation mechanism have been proposed. The study has led to three important conclusions.

1) The TM- and fragment-adaptation strategies are both beneficial for increasing the link throughput and channel utilization. Fragment adaptation can also improve the queuing behavior and, therefore, is more efficient to reduce both PDR and delay.

2) The fragments of a packet may need to be imported through multiple bursts, and thus, more bursts are required to deliver the whole packet. However, the transmission delay increment is marginal compared with the reduced queuing delay.

3) The TM- and fragment-adaptation strategies alone cannot ensure system performance under bad channel conditions, due to their limited adaptivity. The proposed suboptimal joint-adaptation strategy can effectively combat the channel fading and provide the highest link throughput given delay.

\section{REFERENCES}

[1] C. Hu, H. Kim, J. Hou, D. Chi, and S. S. Nandagopalan, "Provisioning quality controlled medium access in ultrawideband-operated WPANs," in Proc. IEEE INFOCOM, Barcelona, Spain, Apr. 2006.

[2] High Rate Ultra Wideband PHY and MAC Standard, ECMA Int. Std. ECMA-368, Dec. 2005.

[3] Wireless Medium Access Control (MAC) and Physical Layer (PHY) Specifications for High Rate Wireless Personal Area Networks (WPANs), IEEE Std. 802.15, Sep. 2003.

[4] M. F. Neuts, J. Guo, M. Zukerman, and H. L. Vu, "Delay analysis for a finite buffer TDMA model," in Proc. IEEE ICC, Anchorage, AK, May 2003, pp. 1791-1795.

[5] M. F. Neuts, J. Guo, M. Zukerman, and H. L. Vu, "The waiting time distribution for a TDMA model with a finite buffer," in Proc. IEEE INFOCOM, San Francisco, CA, 2003, pp. 177-185.

[6] H. Wu, Y. Xia, and Q. Zhang, "Delay analysis of DRP in MBOA UWB MAC," in Proc. IEEE ICC, Istanbul, Turkey, Jun. 2006, pp. 229-233. 
[7] K. H. Liu, X. Shen, R. Zhang, and L. Cai, "Performance analysis of distributed reservation protocol for UWB based WPAN," IEEE Trans. Veh. Technol., vol. 58, no. 2, pp. 902-913, Feb. 2009.

[8] Y. Xiao, X. Shen, and H. Jiang, "Optimal ACK mechanisms of the IEEE 802.15.3 MAC for ultra-wideband systems," IEEE J. Sel. Areas Commun., vol. 24, no. 4, pp. 836-842, Apr. 2006.

[9] H. Chen, Z. Guo, R. Yao, X. Shen, and Y. Li, "Performance analysis of delayed acknowledgement scheme in UWB based high rate WPAN," IEEE Trans. Veh. Technol., vol. 54, no. 2, pp. 606-621, Mar. 2006.

[10] K. H. Liu, H. Rutagemwa, X. Shen, and J. W. Mark, "Efficiency and goodput analysis of Dly-ACK in IEEE 802.15.3," IEEE Trans. Veh. Technol., vol. 56, no. 6, pp. 3888-3898, Nov. 2007.

[11] Q. Liu, S. Zhou, and G. B. Giannakis, "Queuing with adaptive modulation and coding over wireless links: Cross-layer analysis and design," IEEE Trans. Wireless Commun., vol. 4, no. 3, pp. 1142-1153, May 2005.

[12] X. Wang, Q. Liu, and G. Giannakis, "Analyzing and optimizing adaptive modulation coding jointly with ARQ for QoS-guaranteed traffic," IEEE Trans. Veh. Technol., vol. 56, no. 2, pp. 710-720, Mar. 2007.

[13] Multi-Band OFDM Physical Layer Proposal for IEEE 802.15 Task Group 3a, Mar. 2004. IEEE P802.15.3a Working Group, P802.15-03/268r3.

[14] P. Pagani and P. Pajusco, "Characterization and modeling of temporal variations on an ultrawideband radio link," IEEE Trans. Antennas Propag., vol. 54, no. 11, pp. 3198-3206, Nov. 2006.

[15] Z. Irahhauten, J. Dacuna, G. J. Janssen, and H. Nikookar, "UWB channel measurements and results for wireless personal area networks applications," in Proc. Eur. Conf. Wireless Technol., Paris, France, Oct. 2005, pp. $189-192$.

[16] R. Zhang and L. Cai, "A packet-level model for UWB channel with people shadowing process based on angular spectrum analysis," IEEE Trans. Wireless Commun., vol. 8, no. 8, pp. 4048-4055, Aug. 2009.

[17] R. Zhang, L. Cai, S. He, X. Dong, and J. Pan, "Modeling, validation and performance evaluation of body shadowing effect in ultra-wideband networks," Phys. Commun., vol. 2, no. 4, pp. 237-247, Dec. 2009.

[18] R. Zhang and L. Cai, "Analysis of delayed acknowledgment scheme with packet fragmentation of UWB-based WPAN," in Proc. IEEE GLOBECOM, New Orleans, LA, Nov. 2008.

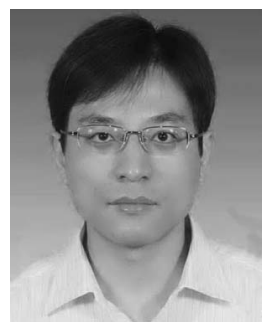

Ruonan Zhang (S'09) received the B.S. and M.S. degrees in electrical engineering from Xi' an Jiaotong University, Xi' an, China, in 2000 and 2003, respectively. He is currently working toward the Ph.D. degree with the Department of Electrical and Computer Engineering, University of Victoria, Victoria, BC, Canada.

$\mathrm{He}$ was with Motorola Inc. and later with Freescale Semiconductor Inc., Tianjin, China, from 2003 to 2006, working on integrated circuit architecture and application design. His current research interests include wireless channel modeling, cross-layer design for wireless networks, and multimedia streaming over wireless networks.

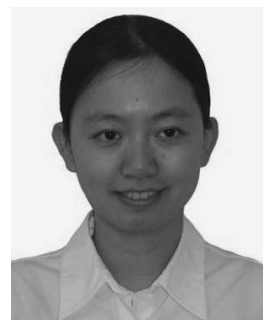

Lin Cai (S'00-M'06) received the M.A.Sc. and $\mathrm{Ph} . \mathrm{D}$. degrees (with an Outstanding Achievement in Graduate Studies Award) in electrical and computer engineering from the University of Waterloo, Waterloo, ON, Canada, in 2002 and 2005, respectively.

Since July 2005, she has been an Assistant Professor with the Department of Electrical and Computer Engineering, University of Victoria, Victoria, BC, Canada. Her research interests span several areas in wireless communications and networking, with a focus on network protocol and architecture design supporting emerging multimedia traffic over wireless, mobile, ad hoc, and sensor networks.

Dr. Cai has been serving as an Associate Editor for the IEEE Transactions on Vehicular TeChNOlogy since 2007, the EURASIP Journal on Wireless Communications and Networking since 2006, and the International Journal of Sensor Networks since 2006. 